Original Article

\title{
Comparison of foot kinetics and kinematics during gait initiation between young and elderly participants
}

\author{
Yoshinao SAtoh, RPT, MS ${ }^{1)^{*}}$, TAKumi Yamada, RPT, PhD ${ }^{2)}$, Ryota Shimamura, RPT, MS ${ }^{1)}$ \\ TAKEHIRo OHMI RPT, MS ${ }^{3}$ \\ 1) Tokyo Metropolitan Rehabilitation Hospital: 2-14-1 Tsutsumidori, Sumida-ku, Tokyo 131-0034, \\ Japan \\ 2) Tokyo Metropolitan University, Japan \\ 3) Clinical Center for Sports Medicine and Sports Dentistry, Tokyo Medical and Dental University, \\ Japan
}

\begin{abstract}
Purpose] To investigate the differences in foot kinetics during gait initiation between young and elderly participants using a modified multi-segment foot model. [Participants and Methods] Twelve young (23.3 \pm 2.4 years $)$ and 12 elderly participants $(73.3 \pm 3.9$ years $)$ were included in this study. Gait initiation was measured using a three-dimensional motion analysis system. We calculated the kinetic and kinematic values using our modified multi-segment foot model and compared those values with the values calculated using Bruening et al.'s multisegment foot model. Modified gait initiation values were also compared between the elderly and young participants. [Results] Our modified multi-segment foot model, created using the Software for Interactive Musculoskeletal Modeling, showed similar values to those reported by Bruening et al. When we compared gait initiation between the elderly participants and their younger counterparts, the elderly exhibited lower torque and power values in the ankle, tarsometatarsal, and metatarsophalangeal joints. Additionally, the elderly exhibited a lower torque ratio in the distal joint than in the proximal joint (torque ratio: ankle joint $>$ tarsometatarsal joint $>$ metatarsophalangeal joint). [Conclusion] The elderly participants had less speed, stride, foot joint movement, moment, and power than the young participants. Moreover, the ratio of joint moment was smaller in the elderly participants. In elderly patients whose walking speed has decreased, consideration of the kinetics of the foot is important when deciding physiotherapy intervention.

Key words: Multi-segment foot model, Gait initiation in elderly, Foot kinetics
\end{abstract}

(This article was submitted Jan. 8, 2019, and was accepted Apr. 3, 2019)

\section{INTRODUCTION}

In a behavior analysis using a three-dimensional motion analysis device, measurements have been performed using the Single-segment Foot Model (SFM). However, in recent years, given the advancement in technology, capturing the position and movement of reflective markers in more detail has been possible. Therefore, the detailed motion analysis of the human body has also become possible.

The Multi-segment Foot Model (MFM) has been developed to analyze the joint motion of the foot. The Oxford Foot Model (OFM) divided the foot into the following three segments: hind foot, metatarsal part, and toe parts. OFM allows the kinematic analysis between the foot segments. Carson et al. ${ }^{1)}$, Stebbins et al. ${ }^{2}$, and Curtis et al. ${ }^{3)}$ have reported OFM reliability. However, OFM cannot analyze the kinetics, such as moment and power, of the foot ${ }^{1-3)}$.

*Corresponding author. Yoshinao Satoh (E-mail: satouyoshinao2000@yahoo.co.jp)

(C2019 The Society of Physical Therapy Science. Published by IPEC Inc.

(c) (1) $\odot$ This is an open-access article distributed under the terms of the Creative Commons Attribution Non-Commercial No Derivatives cc) 
In general, the elderly has a slower walking speed and lesser stride length than young individuals. The slower walking speed is thought to be related to the deterioration of the control function in the single leg supporting phase, extension of the double support phase, and decrease in stride length ${ }^{4}$.

In terms of kinematic feature, the walk of the elderly is characterized by a decreased maximum extension angle of the hip joint ${ }^{5)}$, and decreased maximum dorsiflexion and plantarflexion angles of the ankle joint ${ }^{6}$. Moreover, in recent kinematic studies using MFM, Legault-Moore et al. ${ }^{7}$ ) reported that there was no significant difference in the joint movement of the foot between the young (24.6 \pm 3.0 years) and elderly (65.0 \pm 4.2 years) participants. On the other hand, Arnold et al. reported that the elderly had decreased ankle, midfoot-calcaneus, and hallux-metatarsus joint movement on the sagittal plane, and metatarsus-midfoot joint movement on the coronal plane ${ }^{8)}$, which could be due to the influence of age, as the participants were of varying ages. In kinematic analysis using SFM, it is reported that the elderly had decreased ankle joint plantar flexion moment and positive power ${ }^{6,9)}$. Bruening et al.'s MFM divided the foot into the following three segments: toes, metatarsal part, and hind foot; they reported foot kinematics and kinetics ${ }^{10,11)}$. Additionally, they reported that the SFM and MFM have different ankle kinetics; because of differences in ankle joint movement between the two models, the SFM overestimates ankle power ${ }^{10,11)}$. In considering the walking problem with aging, the influence of the foot cannot be ignored. However, no studies have compared the kinetics between the elderly and young individuals using the MFM. Therefore, this study aimed to analyze foot kinetics and compare the differences in foot kinetics and kinematics in gait initiation between young and elderly participants using a modified MFM. (a)

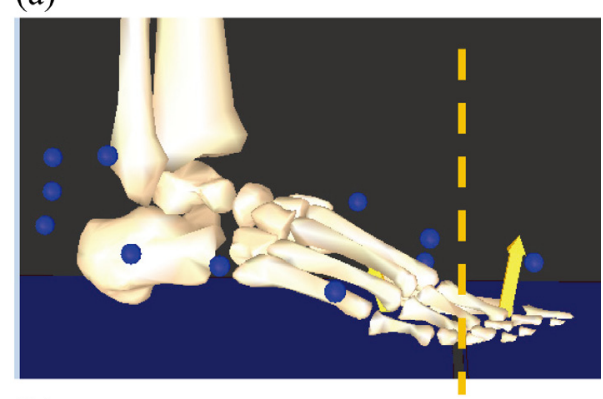

(b)

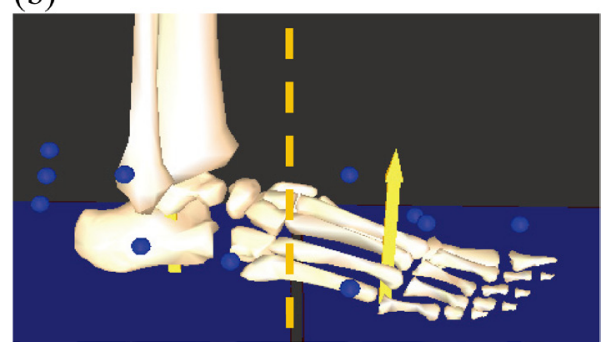

Fig. 1. Starting position.

(a) to analyze toe joint kinetics.

(b) to analyze midfoot joint kinetics.

\section{PARTICIPANTS AND METHODS}

Twelve healthy young ( 5 males, 7 females, age: $23.3 \pm 2.4$ years, height: $164.6 \pm 7.8 \mathrm{~cm}$, body weight: $56.1 \pm 7.1 \mathrm{~kg}$ ) and 12 elderly participants ( 6 males, 6 females, age: $73.3 \pm 3.9$ years, height: $155.9 \pm 11.3 \mathrm{~cm}$, body weight: $55.1 \pm 9.4 \mathrm{~kg}$ ) living in the area participating in the health promoting program were recruited for this study. All participants had no orthopedic or neurological disability and were able to walk without using walking aids.

We measured height, weight, leg length, knee joint width, ankle joint width, foot length, foot width, foot circumference, arch length, arch height, and arch height ratio (arch height/arch length) of all participants. Then, we measured gait initiation using a three-dimensional motion analysis system with 10 infrared cameras (Vicon Nexus; Oxford Metrics, London, UK).

Thirty-three infrared retroreflective markers were attached to various anatomical locations according to the OFM marker set (anterior superior iliac spine, posterior superior iliac spine, sacrum, outside of the thigh, femoral lateral condyle, femoral medial condyle, front side of the shank, outside of the shank, fibular head, tibial rough surface, tibial rough surface, lateral malleolus, medial malleolus, calcaneus protuberance, proximal part of the calcaneus, Achilles tendon attachment part, outside of the calcaneus, sustentaculum tali, first metatarsal bone bottom, first metatarsal head, fifth metatarsal bone bottom, fifth metatarsal head, second metatarsal head, and hallux).

The ground reaction forces were captured using four Kisler force plates (Kisler Japan, Tokyo, Japan). The forces were measured using two adjacent force plates on a foot to analyze midfoot and toe joint kinetics as described in a previous study ${ }^{11)}$. We chose gait initiation because it allows the measurement of 2 ground reaction forces (GRFs) separately between the foot segments. The speed and stride in gait initiation were arbitrary by the participants and were not stipulated.

The MFM divided the foot part into the following three segments: the toe part, the metatarsal part, the hind foot part (Fig. 1). The Software for Interactive Musculoskeletal Modeling (SIMM; MusculoGraphics, Santa Rosa, CA, USA) was used to analyze foot kinematics and kinetics. In the MFM, the joints were placed between the metatarsal and hind foot parts (MHJ), and between the toe and metatarsal parts (TMJ). We smoothed the measurement data using the Gap Filling and Butter Worth Filter on the Vicon Nexus. Then, the measurement data were loaded into the SIMM. The joint angle was calculated by using the Vicon Nexus (OFM) and SIMM, whereas joint moment and power were calculated by using the SIMM. In addition, joint moment and power were divided according to the participants' body weights to be normalized.

Data are expressed as mean \pm standard deviation. The joint angle of the foot was calculated using OFM and SIMM based on the same trial data. Subsequently, Pearson's correlation coefficient was used to analyze the difference between their values. An unpaired t-test was performed to analyze the following physical measurement parameters and gait parameters of the elderly and young participants. Physical measurement parameters were height, weight, leg length, knee joint width, ankle 
Table 1. Correlation of values calculated for OFM and SIMM

\begin{tabular}{llcc}
\hline & $\mathrm{r}=$ & OFM $\left(^{\circ}\right)$ & SIMM $\left(^{\circ}\right)$ \\
\hline AJ dorsal flexion & $0.77^{* *}$ & $16.9 \pm 3.9$ & $16.3 \pm 3.5$ \\
AJ plantar flexion & $0.95^{* *}$ & $11.7 \pm 8.0$ & $5.9 \pm 8.2$ \\
AJ range & $0.92^{* *}$ & $28.1 \pm 7.9$ & $22.2 \pm 8.0$ \\
MHJ dorsal flexion & $0.70^{* *}$ & $19.2 \pm 4.9$ & $10.5 \pm 4.3$ \\
MHJ plantar flexion & 0.32 & $3.4 \pm 5.4$ & $3.2 \pm 3.1$ \\
MHJ range & $0.46^{*}$ & $22.6 \pm 5.9$ & $13.7 \pm 3.1$ \\
TMJ dorsal flexion & $0.92^{* *}$ & $27.5 \pm 9.8$ & $33.4 \pm 12.6$ \\
TMJ plantar flexion & $0.76^{* *}$ & $12.3 \pm 8.5$ & $6.9 \pm 6.4$ \\
TMJ range & $0.91^{* *}$ & $39.9 \pm 12.2$ & $40.3 \pm 13.2$ \\
\hline
\end{tabular}

$\mathrm{r}=$ Pearson's Correlation coefficient, $* * \mathrm{p}<0.01,{ }^{*} \mathrm{p}<0.05$.

AJ: Ankle joint; MHJ: metatarsal-hind foot joint; TMJ: toe-metatarsal joint.

Correlation of the values calculated by the two models based on the same trial data.

Table 2. Physical measurement parameter of elderly and young participants

\begin{tabular}{lcclcc}
\hline & Young & Elderly & & Young & Elderly \\
\hline Age (years) & $23.3 \pm 2.4$ & $73.3 \pm 3.9$ & Foot length $(\mathrm{cm})$ & $24.3 \pm 1.2$ & $23.4 \pm 1.7$ \\
Body weight $(\mathrm{kg})$ & $56.1 \pm 7.1$ & $55.1 \pm 9.4$ & Arch length $(\mathrm{cm})$ & $18.8 \pm 1.1$ & $18.1 \pm 1.4$ \\
Height $(\mathrm{cm})$ & $164.6 \pm 7.8$ & $156.0 \pm 11.3$ & Arch height $(\mathrm{cm})$ & $6.2 \pm 0.6$ & $6.7 \pm 0.6$ \\
Leg length $(\mathrm{cm})$ & $83.3 \pm 3.8$ & $72.4 \pm 2.2$ & Foot width $(\mathrm{cm})$ & $9.5 \pm 0.8$ & $9.7 \pm 0.7$ \\
Knee width $(\mathrm{cm})$ & $9.8 \pm 0.6$ & $9.7 \pm 0.7$ & Foot circumference $(\mathrm{cm})$ & $23.2 \pm 1.6$ & $23.8 \pm 1.8$ \\
Ankle width $(\mathrm{cm})$ & $6.7 \pm 0.5$ & $6.8 \pm 0.7$ & Arch height ratio (arch height/arch length) & $0.3 \pm 0.02$ & $0.4 \pm 0.03$ \\
\hline
\end{tabular}

There were no significant differences between young people and the elderly in all physical measurement parameters.

joint width, foot length, foot width, foot circumference, arch length, arch height, and arch height ratio. Gait parameters were walking speed, stride length, peak angle (plantar flexion, dorsal flexion), peak moment (plantar flexion), and peak power of the ankle joint (AJ), MHJ (between metatarsal and hind foot joint), and TMJ (between toe and metatarsal joint). Moreover, to compare the motion ratios of $\mathrm{AJ}, \mathrm{MHJ}$, and TMJ during gait initiation, an unpaired t-test was performed for the ratio of the movement range, joint moment, and joint power of MHJ/AJ, TMJ/AJ, and TMJ/MHJ. IBM SPSS Statistics version 22 was used for all statistical analyses, and the significance level was set at $5 \%$.

The study protocol adhered to the guidelines of the Declaration of Helsinki and was approved by the Tokyo Research Safety Ethics Committee of the Tokyo Metropolitan University (Approval No. 13082). In addition, the study participants were fully informed of the content and purpose of the research, and the study procedures were carried out after obtaining written consent from the participants.

\section{RESULTS}

For the OFM, the range of joint motion was as follows: AJ, $28.1 \pm 7.9^{\circ}$; MHJ, 22.6 $\pm 5.9^{\circ}$; and TMJ, $39.9 \pm 12.2^{\circ}$. For the SIMM, the range of joint motion was as follows: AJ, $22.2 \pm 8.0^{\circ}$; MHJ, $13.7 \pm 3.1^{\circ}$; and TMJ, $40.3 \pm 13.0^{\circ}$. The correlation coefficients between OFM and SIMM for AJ dorsiflexion, AJ plantarflexion, AJ movement range, MHJ dorsiflexion, MHJ plantar flexion, MHJ movement range, TMJ extension, TMJ flexion, and TMJ movement range were 0.77, 0.95, 0.91, 0.70, $0.32,0.46,0.92,0.76$, and 0.91 , respectively (Table 1 ).

There was no significant difference in all physical measurement parameters between the elderly and young participants (Table 2). The walking speed was significantly lower in the elderly than in the young participants $(\mathrm{p}<0.05)$. The elderly had a significantly lower stride length value compared to the young participants $(p<0.05)$. The elderly participants had a significantly lower mean angle compared to the young participants $(\mathrm{p}<0.05)$. The mean peak AJ dorsal flexion, MHJ plantar flexion, and TMJ plantar and dorsal flexion angles were not significantly different between the young and elderly participants. The elderly participants had a significantly lower mean angle than the young participants $(\mathrm{p}<0.05)$. The mean TMJ movement range was not significantly different between the young and elderly participants. The mean ratios of MHJ/AJ, TMJ/AJ, and $\mathrm{TMJ} / \mathrm{MHJ}$ movement ranges were not significantly different between the young and elderly participants (Table 3).

The mean peak values of the AJ plantar flexion moments were $1.45 \pm 0.14 \mathrm{Nm} / \mathrm{kg}$ and $1.20 \pm 0.20 \mathrm{Nm} / \mathrm{kg}$ for the younger and elderly participants, respectively. The mean MHJ plantar flexion moment peak values were $0.76 \pm 0.08 \mathrm{Nm} / \mathrm{kg}$ and 0.60 $\pm 0.08 \mathrm{Nm} / \mathrm{kg}$ for the younger and elderly participants, respectively. The mean TMJ plantar flexion moment peak values were $0.12 \pm 0.04 \mathrm{Nm} / \mathrm{kg}$ and $0.07 \pm 0.03 \mathrm{Nm} / \mathrm{kg}$ for the younger and elderly participants, respectively. The elderly had significantly lower mean plantar flexion moment peak values than the young participants $(\mathrm{p}<0.05)$. The ratio of MHJ/AJ joint moment was $52.3 \pm 3.3 \%$ and $47.5 \pm 3.9 \%$ for the younger and elderly participants, respectively. The TMJ/AJ joint moment ratio was 8.6 
Table 3. Kinematics parameter of elderly and young participants

\begin{tabular}{lccc}
\hline & Young & Elderly & \\
\hline Gait speed $(\mathrm{m} / \mathrm{sec})$ & $0.80 \pm 0.08$ & $0.60 \pm 0.15$ & $*$ \\
Step length $(\%)$ & $74.9 \pm 8.1$ & $58.6 \pm 11.2$ & $*$ \\
Joint motion $\left({ }^{\circ}\right)$ & & & \\
AJ dorsal flexion & $16.0 \pm 4.1$ & $15.8 \pm 4.1$ & \\
AJ plantar flexion & $10.8 \pm 6.9$ & $1.0 \pm 6.2$ & $*$ \\
AJ range & $26.8 \pm 7.4$ & $16.7 \pm 4.1$ & $*$ \\
MHJ dorsal flexion & $13.4 \pm 5.1$ & $8.5 \pm 4.4$ & $*$ \\
MHJ plantar flexion & $2.5 \pm 3.0$ & $2.9 \pm 4.0$ & $*$ \\
MHJ range & $16.0 \pm 3.4$ & $11.4 \pm 3.0$ & \\
TMJ dorsal flexion & $35.8 \pm 13.9$ & $31.7 \pm 12.4$ & \\
TMJ plantar flexion & $4.5 \pm 6.3$ & $2.4 \pm 7.8$ & \\
TMJ range & $40.4 \pm 12.9$ & $34.2 \pm 13.3$ & \\
Ratio of range $(\%)$ & & & \\
MHJ/AJ & $64.0 \pm 21.9$ & $71.6 \pm 25.4$ & \\
TMJ/AJ & $165.2 \pm 78.9$ & $206.0 \pm 86.3$ & \\
TMJ/MHJ & $266.4 \pm 97.8$ & $290.4 \pm 93.7$ & \\
\hline
\end{tabular}

$* \mathrm{p}<0.05$.

AJ: Ankle joint; MHJ: metatarsal-hind foot joint; TMJ: toe-metatarsal joint.

Table 4. Kinetics parameter of elderly and young participants

\begin{tabular}{lccc}
\hline Plantar flexion moment $(\mathrm{Nm} / \mathrm{kg})$ & Young & Elderly & \\
\hline AJ & $1.45 \pm 0.13$ & $1.20 \pm 0.21$ & $*$ \\
MHJ & $0.75 \pm 0.08$ & $0.60 \pm 0.08$ & $*$ \\
TMJ & $0.12 \pm 0.04$ & $0.07 \pm 0.03$ & $*$ \\
Ratio of moment (\%) & & & \\
MHJ/AJ & $52.3 \pm 3.3$ & $47.5 \pm 4.0$ & $*$ \\
TMJ/AJ & $8.7 \pm 2.9$ & $5.9 \pm 2.2$ & $*$ \\
TMJ/MHJ & $16.5 \pm 5.2$ & $12.1 \pm 4.7$ & $*$ \\
Power (Watts/kg) & & & \\
AJ positive & $2.07 \pm 0.64$ & $1.02 \pm 0.43$ & $*$ \\
MHJ positive & $0.90 \pm 0.19$ & $0.54 \pm 0.24$ & $*$ \\
TMJ negative & $0.42 \pm 0.14$ & $0.28 \pm 0.12$ & $*$ \\
Ratio of power (\%) & & & \\
MHJ/AJ & $46.6 \pm 13.3$ & $53.9 \pm 10.7$ & \\
TMJ/AJ & $23.4 \pm 14.8$ & $28.4 \pm 8.3$ & \\
TMJ/MHJ & $49.7 \pm 23.2$ & $53.7 \pm 15.2$ & \\
\hline
\end{tabular}

$* \mathrm{p}<0.05$.

AJ: Ankle joint; MHJ: metatarsal-hind foot joint; TMJ: toe-metatarsal joint.

$\pm 2.9 \%$ and $5.9 \pm 2.2 \%$ for the younger and elderly participants, respectively. The TMJ/MHJ joint moment ratio was $16.5 \pm$ $5.2 \%$ and $12.1 \pm 4.7 \%$ for the younger and elderly participants, respectively. The elderly had significantly lower joint moment ratios compared to the young participants $(\mathrm{p}<0.05)$.

The mean AJ positive power was $2.07 \pm 0.64 \mathrm{~W} / \mathrm{kg}$ and $1.02 \pm 0.42 \mathrm{~W} / \mathrm{kg}$ for the younger and elderly participants, respectively. The mean MHJ positive power was $0.90 \pm 0.20 \mathrm{~W} / \mathrm{kg}$ and $0.54 \pm 0.24 \mathrm{~W} / \mathrm{kg}$ for the younger and elderly participants, respectively. The mean TMJ negative power was $0.42 \pm 0.14 \mathrm{~W} / \mathrm{kg}$ and $0.28 \pm 0.12 \mathrm{~W} / \mathrm{kg}$ for the younger and elderly participants, respectively. The elderly had significantly lower positive or negative power values compared to the young participants $(\mathrm{p}<0.05)$. The ratios of $\mathrm{MHJ} / \mathrm{AJ}, \mathrm{TMJ} / \mathrm{AJ}$, and TMJ/MHJ joint power did not differ significantly between the elderly and young participants (Table 4).

\section{DISCUSSION}

In this study, we evaluated the differences in foot kinetics during gait initiation between young and elderly participants using the MFM. Our findings revealed that foot kinetics were calculated using the MFM created on the SIMM. Moreover, in terms of foot kinematics, the elderly had lesser speed, stride, foot joint movement, moment, and power than the young participants. Additionally, the elderly exhibited lower torque ratio of the distal joint than of the proximal joint.

The AJ and TMJ angles showed high correlation coefficients among the OFM and SIMM, indicating that there was no obvious difference between the models in these joints. With regard to MHJ angle, the correlation coefficient was low. In addition, the average range of motion of MHJ significantly differ between OFM and SIMM (22.6 and $13.7^{\circ}$, respectively). This 
angle difference may be due to the differences in the calculation process among the models. Bruening et al.' $\mathrm{s}^{10)}$ MFM showed that the range of motion of the dorsiflexion of MHJ in the stance phase of young people was about $13^{\circ}$ (range $14^{\circ}-27^{\circ}$ ), which was equivalent to the range of motion calculated using SIMM. Therefore, we considered that the MFM created by using SIMM could analyze the joint motion with results closely similar to those of Bruening et al.'s MFM, in comparison to OFM.

In the kinetics of the foot part in young people, Bruening et al. ${ }^{11)}$ reported that the plantar flexion moment of AJ, MHJ, and TMJ was approximately $1.2,0.9$, and $0.1 \mathrm{Nm} / \mathrm{kg}$, respectively, and their power was $2.1,1.2$, and $0.5 \mathrm{~W} / \mathrm{kg}$, respectively, which were closely similar to the results of the young participants in our study. Thus, we considered that our MFM created by using SIMM had a high validity.

In the physical measurement parameters, there were no significant differences between the young and elderly participants in the physical measurement parameters. In Faria et al.'s study ${ }^{12)}$ involving 81 postmenopausal females (age $58.0 \pm 6.0$ years), the arch structure showed a moderate correlation with the body mass index $(\mathrm{r}=0.554)$ and poor correlation with age $(\mathrm{r}=0.260)$. Given that age and arch structure were poorly correlated, and this study had a small sample size, we considered that there were no significant differences between the young and elderly participants in terms of physical measurement parameters.

We decided to analyze gait initiation rather than normal walking, because this allowed us to measure 2 GRFs separately between two foot segments: the toe and metatarsal part, and the metatarsal part and hind foot. In the study of Naramoto et al. ${ }^{13)}$, which compared normal gait and gait initiation, the locus of the center of the foot pressure showed a similar pattern, and no statistically significant difference was found, except for the heel contact period. Therefore, we considered that gait initiation was equal to normal gait at the later stage of the stance phase, which is the peak of the plantar flexion movement.

In this study, the walking speed, stride length, AJ plantar flexion angle, AJ movement range, MHJ dorsiflexion angle, MHJ movement range, and AJ positive power were lower in the elderly than in the younger participants. In addition, this study also showed that MHJ plantar flexion moment, TMJ plantar flexion moment, MHJ positive power, TMJ negative power, and the ratio of joint moments $(\mathrm{MHJ} / \mathrm{AJ}, \mathrm{TMJ} / \mathrm{AJ}$, and $\mathrm{TMJ} / \mathrm{MHJ})$ of the elderly were lower compared to those of the younger participants. As mentioned below, decreased walking speed and stride length of the elderly might be affected by the decreased joint moments and powers not only of the ankle joint but also of the foot and toe. The decreased moments seemed to occur from the toes.

Scott et al. ${ }^{14)}$ reported that the elderly had decreased foot toe compressive muscular strength and decreased walking speed and stride. In foot pressure measurement, they also reported that the maximum pressure of the calcaneus, lateral metatarsal, and hallucination parts decreased in the elderly. From results of this study and previous reports, elderly people seemed to have lower mechanical support in the toes and lower stride, which could be possibly due to the decrease in the compressive muscular strength of the toes.

The limitation of this study was to analyze the walking gait initiation from the standing position to measure the GRFs separately between the foot segments. Therefore, unlike normal gait, it was not possible to examine the foot function in the first half of the stance phase. Both the elderly and young participants had narrow age ranges.

Because it seems that examining the relationship between foot kinematics and other lower limb joints such as the knee and hip joints is important for the elderly walking problem, future research will be necessary.

In conclusion, this study demonstrated that MFM created using SIMM could be used to calculate the foot kinetics. Comparing the foot kinematics during gait initiation between the elderly and young participants, the elderly had lesser speed, stride, foot joint movement, moment, and power than the young participants. Moreover, the elderly exhibited lower torque ratio of the distal joint than of the proximal joint. The elderly may possibly have weakened moment from the toe. Consideration of the kinetics of the foot is important in deciding physiotherapy intervention for elderly patients whose walking speed has decreased.

Funding and Conflict of interest

The authors have no conflicts of interest directly relevant to the content of this article.

\section{ACKNOWLEDGEMENT}

We would like to thank Editage (www.editage.jp) for English language editing.

\section{REFERENCES}

1) Carson MC, Harrington ME, Thompson N, et al.: Kinematic analysis of a multi-segment foot model for research and clinical applications: a repeatability analysis. J Biomech, 2001, 34: 1299-1307. [Medline] [CrossRef]

2) Stebbins J, Harrington M, Thompson N, et al.: Repeatability of a model for measuring multi-segment foot kinematics in children. Gait Posture, 2006, 23: 401-410. [Medline] [CrossRef]

3) Curtis DJ, Bencke J, Stebbins JA, et al.: Intra-rater repeatability of the Oxford foot model in healthy children in different stages of the foot roll over process during gait. Gait Posture, 2009, 30: 118-121. [Medline] [CrossRef] 
4) Rose J, Gamble J: Human walking. Tokyo: Ishiyakushuppan, 2009, pp 141-147.

5) Kerrigan DC, Todd MK, Della Croce U, et al.: Biomechanical gait alterations independent of speed in the healthy elderly: evidence for specific limiting impairments. Arch Phys Med Rehabil, 1998, 79: 317-322. [Medline] [CrossRef]

6) DeVita P, Hortobagyi T: Age causes a redistribution of joint torques and powers during gait. J Appl Physiol 1985, 2000, 88: 1804-1811. [Medline] [CrossRef]

7) Legault-Moore D, Chester VL, de Vries G: Multisegment foot kinematics during walking in younger and older adults. J Clin Med Res, 2012, 4: 259-266. [Medline]

8) Arnold JB, Mackintosh S, Jones S, et al.: Differences in foot kinematics between young and older adults during walking. Gait Posture, 2014, 39: 689-694. [Medline] [CrossRef]

9) Anderson DE, Madigan ML: Healthy older adults have insufficient hip range of motion and plantar flexor strength to walk like healthy young adults. J Biomech, 2014, 47: 1104-1109. [Medline] [CrossRef]

10) Bruening DA, Cooney KM, Buczek FL: Analysis of a kinetic multi-segment foot model. Part I: Model repeatability and kinematic validity. Gait Posture, 2012, 35: 529-534. [Medline] [CrossRef]

11) Bruening DA, Cooney KM, Buczek FL: Analysis of a kinetic multi-segment foot model part II: kinetics and clinical implications. Gait Posture, 2012, 35: 535-540. [Medline] [CrossRef]

12) Faria A, Gabriel R, Abrantes J, et al.: The relationship of body mass index, age and triceps-surae musculotendinous stiffness with the foot arch structure of postmenopausal women. Clin Biomech (Bristol, Avon), 2010, 25: 588-593. [Medline] [CrossRef]

13) Naramoto S, Ogawa T: Changes in foot pressure center trajectory at walking start and normal walking. Rigakuryouhou-sinnpo-to-tennbou, 2007, 21: 16-22 (in Japanese).

14) Scott G, Menz HB, Newcombe L: Age-related differences in foot structure and function. Gait Posture, 2007, 26: 68-75. [Medline] [CrossRef] 